\title{
BÜROKRATIE
}

\section{Unsere heimliche Geliebte}

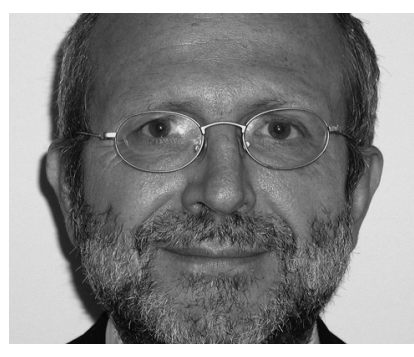

VON WOLFGANG GRATZ Univ.Prof. Dr. Mag. Wolfgang Gratz ist Jurist, Soziologe, habilitierter Kriminologe und arbeitet als Studiengangsleiter Public Management an der FH Campus Wien. Zudem ist er freiberuflich als Berater und Trainer tätig. Zuvor war er in langjährigen Leitungstätigkeiten im Strafvollzug und bei der österreichischen Bewährungshilfe (nunmehr "Neustart») engagiert.

E-Mail wolfgang.gratz@fh-campuswien.ac.at
Für die Verantwortlichen von Ämtern und Unternehmen ist Bürokratie attraktiv, weil sie es den Führungskräften ermöglicht, sich als Teil der Lösung und nicht als Teil des Problems anzusehen. Soziale Dienstleister sollten deshalb ihr Organigramm einfach umdrehen: Ganz oben sollten die Organisationseinheiten stehen, die unmittelbar mit dem Klienten arbeiten.

Wie Max Weber herausarbeitete, bedeutet Bürokratie die Eindämmung persönlicher Willkür, die Gewährleistung rationaler Herrschaft. Die Organisation menschlicher Arbeit nach einem ähnlichen Maschinenmodell als Fließbandarbeit in der Industrie (Taylorismus) ermöglichte Massenproduktion als Basis des Lebensstandards, den wir genießen. Die Organisation der Erbringung von Leistungen durch genaue Festlegung von Vorgehensweisen und Ergebnissen sowie durch Aufspaltung komplexer Abläufe in kleine Arbeitspakete kann man auch als zivilisatorischen Fortschritt ansehen.

Bürokratie ist keineswegs auf den öffentlichen Sektor begrenzt. Um ein Beispiel zu geben: Eine Kollegin erzählte mir, dass sie für eine Dienstreise als Beamtin eine Unterschrift benötigte, als Consulterin in einem global agierenden Konzern vier Unterschriften. Es gibt Checklisten für die Bewertung des Personalverhaltens im FastFoodbereich, die für die Bedienung eines einzelnen Kunden mehr als 30 Teilaspekte beinhalten.

Es gibt einerseits Bürokratie im alten Gewand der Vorschriften, andererseits Bürokratie im modisch-schicken Outfit von Qualitätsmanagement, Risikomanagement, Ziel- und Leistungsvereinbarungen, Controlling, Prozessbeschreibungen und dergleichen mehr. Teilweise ist Projektmanagement, das die Limitierungen und Defizite formalisierter Linienorganisationen überwinden soll, seinerseits zu einem bürokratischen Klon geworden. Auf diese Weise entstanden eine Reihe neuer Professionen mit zertifizierten und akademisierten Ausbildungen (Qualitätsmanager, Controller, Projektmanager ...). Diese Techniken haben ebenso das Potenzial, unternehmerisches Handeln zu begünstigen und zu professionalisieren wie es zu ersticken.
Neue und alte Bürokratien setzen den tief liegenden Ängsten vor Chaos und Kontrollverlust den Mythos entgegen, dass die Menschen, ihre Interaktionen und das Dasein an sich beherrschbar seien, wenn denn alles genügend geregelt wäre.

Organisationsmodelle, die auf Selbstorganisation, Vertrauen und ökologischen Prinzipien beruhen, sind hingegen exponierte Biotope, von denen man sich bei auftretenden Problemen rasch wieder trennt. Man kehrt dann zu den vertrauten bürokratischen Monokulturen zurück. Andererseits funktionieren Bürokratien bei Problemen nach dem Grundsatz: mehr vom selben, also mehr Kontrolle und Normierung. Sie stellen somit hochstabile Systeme dar.

Ein wichtiger Erfolgsfaktor von Bürokratie liegt aber auch in uns selbst. Die bürokratische Hölle sind nicht nur die anderen. Wer ist schon gänzlich frei vom Bedürfnis, dass Menschen und Dinge sich genau so und nicht anders verhalten als es seinen Erwartungen entspricht? Ein solches Kontrollbedürfnis hat jedenfalls in jeder Bürokratenseele seinen festen Platz.

Bürokratie ist aber auch deshalb so attraktiv, weil sie es den Führungskräften ermöglicht, was immer auch geschieht, sich als Teil der Lösung und nicht als Teil des Problems anzusehen. Sie stellen VerschiebeBahnhöfe dar, in denen die Verantwortung über eine Vielzahl von Weichen nach unten rollt, bis sie am »Point of Sale «, also am Ort der Leistungserbringung wie an einem Prellbock hängenbleibt.

Je kundennäher die Mitarbeiterinnen und Mitarbeiter handeln, desto leichter geraten sie mit den bürokratischen Regelungen im Konflikt. Da solche Spannungsfelder nicht offen angesprochen werden (dürfen), bilden die Mitarbeitenden Formen von Gegenmacht aus. Das Spektrum reicht von »die Vorgesetzten dumm sterben lassen « bis zu mehr oder weniger subtilen Widerstandstaktiken.

Auf diese Weise bestätigen sie die in Bürokratien vorherrschenden Glaubenssätze, dass Vorgesetzte sich autoritär durchsetzen müssen, ohne die Situation und Bedürfnisse der Mitarbeiterinnen zu beachten. Man kann in Abwandlung eines Zitates von 
Karl Kraus sagen: Bürokratie ist die Krankheit, für deren Therapie sie sich hält.

Die horizontale und vertikale Zergliederung von Aufgaben führt dazu, dass es keine klare Zuständigkeit für komplexe Aufgabenstellungen gibt. Der Schein-Klarheit des Organigramms steht in der Praxis die organisierte Verantwortungslosigkeit gegenüber.

Bürokratien haben eine Leidenschaft, Daten zu sammeln und eine Abscheu, diese Daten diskursiv zu verwerten.

Bürokratien sind hoch kompetent, auf veränderte Umweltbedingungen so zu reagieren, dass ihre gewohnte Operationslogik nicht infrage gestellt wird. Verschiedentlich führt die Implementierung betriebswirtschaftlicher Instrumente in Bürokratien nicht zu neuer unternehmerischer Identität, sondern zu neuen Formen der Bürokratie.

Bürokratien neigen dazu, Menschen so zu behandeln, als ob sie auf der Entwicklungsstufe vormenschlicher Primaten stehengeblieben wären. Sie negieren intrinsische, also aus der Eigen-Sinnlichkeit und Eigen-Willigkeit von Individuen entspringende Motive und setzen nicht auf Selbstbeobachtung und Reflexion als Voraussetzungen persönlicher und organisationaler Weiterentwicklung.

Bürokratien sind sehr schlecht befähigt, ihr innenbezügliches Eigenleben aufzuge- ben, sich mit anderen Organisationen zu vernetzen und Kooperationen einzugehen. Hierbei spielt ihre Schwerfälligkeit eine zentrale Rolle.

In Bürokratien leidet die Produktivität in hohem Ausmaß durch kommunikative Defizite. Die offiziellen Kommunikationswege sind kompliziert und zeitaufwändig. Der informellen Kommunikation kommt hohe Bedeutung zu. Sie nimmt viel Zeit in Anspruch und ist zumeist mehr von Machtkalkülen und Machtspielen denn von Aufgabenorientierung geprägt. Diese Formen der Vergeudung von Arbeitszeit werden ebenso tabuisiert, wie ineffiziente Besprechungssysteme ein zähes, unhinterfragtes Leben haben.

\section{Das Kegelorganigramm - ein systemischer Vorschlag}

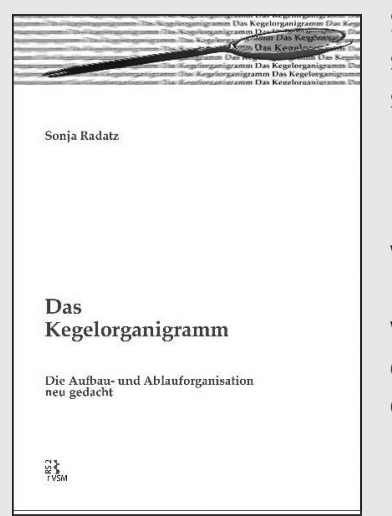

Sonja Radatz, Vorsitzende der Geschäftsführung des Instituts für systemisches Coaching und Training mit Standorten in Wien und Hamburg, beschreibt in ihrem Buch "Das Kegelorganigramm ", wie mit dem Visualierungsinstrument des Organigramms ein bewusst anders gestaltetes Abbild des Unternehmens erzeugt und damit zur Grundlage für Veränderungen werden kann. Auszüge aus ihrem Buch:

"Während herkömmliche Organigramme Hierarchiestufen, Funktionen und Positionen (verbunden mit bestimmten Namen von Mitarbeitern) abbilden, geht es aus konstruktivistischer Sicht um Verhaltensbeiträge - und nicht nur darum, sondern auch um eine ausschließliche Betrachtung des Werts dieser Beiträge unter dem Fokus der gemeinsam formulierten Ziele. Letztendlich ist es unerheblich, von wem im Unternehmen diese Beiträge stammen! Das Organigramm sollte also aus systemisch-konstruktivistischer Sicht nicht Hierarchieunterschiede, sondern Unterschiede in den Verhaltensbeiträgen zeigen.«

"Mit dem Modell des Kegelorganigramms soll eine Antwort auf die systemisch-konstruktivistischen Anforderungen an das Organigramm entwickelt werden:

- es soll prozessorientiert sein, d. h. auf Verhaltensbeiträge fokussieren

- es soll die Kernprozesse des Unternehmens - Reflexion und Anpassung - optimal unterstützen

- es soll bewusst einladen, vielfältige Sichtweisen zu entwickeln

- es soll ermöglichen, die Handlungsalternativen in einem Unternehmen zu vergrößern

Daraus entsteht ein Modell, das jeweils ein Bild des Augenblicks bietet - aus einer subjektiven Sicht. Es dient nicht der Festschreibung, sondern der Anregung von Reflexion und Entscheidung (ob im Dienste der bestehenden Ziele bzw. Herausforderungen Verhaltensbeiträge verändert oder beibehalten werden sollen). Jeder Mitarbeiter eines Unternehmens bzw. einer Orga- nisation hat gleichberechtigt die Möglichkeit, seine Sichtweise in diesem Organigramm darzustellen und damit auch etwas über sich selbst - seine eigenen Erfahrungen - preiszugeben. Diese verschiedenen Sichtweisen erweitern wiederum die Handlungsmöglichkeiten des Unternehmens."

"Das von mir entwickelte Kegelorganigramm stellt einen Bezug zwischen den im Relationsmodell vorhandenen Menschen und der Struktur des Systems im weiteren Sinne dar, indem die Menschen in Form ihrer beeinflussenden Verhaltensbeiträge in die Struktur hereingeholt werden und das Organigramm so ein Zusammenfließen aus Verhaltensbeiträgen und Beitragsebenen - nach innen und nach außen - darstellt. Die für das Unternehmen vorrangig relevanten Unterscheidungen werden dabei in das Modell integriert und bilden so gewissermaßen einen Reflexionsrahmen, in dem Veränderungen angedacht werden können:

- Beiträge auf unterschiedlichen Ebenen (inhaltlicher Fokus)

- Beiträge nach innen vs. nach außen (Wirksamkeitsfokus)

- Autoritätshandeln vs. Teamhandeln (Entstehungs- bzw. Prozessfokus)

Das Kegelorganigramm kann sowohl auf Teamebene (Teamoder Abteilungssystem) als auch auf Organisationsebene (Organisationssystem) verwendet werden.

Das Kegelorganigramm schafft ein subjektives Abbild des Unternehmens und gibt damit auf innovative und vielleicht auch überraschende Weise Antworten auf Fragen wie:

- Inwieweit ist unser Unternehmen unseren Zielen entsprechend nach außen/innen orientiert?

- Passen unsere Entscheidungsformen zu unserer Strategie?

- Wer konzentriert sich im Unternehmen aufdie Arbeit an den Zielen/Strategien, an den Prozessen, Kommunikationen, konkreten Handlungen?

- Wo müssen wir ansetzen, um eine für uns optimale Balance im unternehmensspezifischen Kegelorganigramm zu erreichen?«

Quelle: Sonja Radatz: Das Kegelorganigramm. Die Aufbau- und Ablauforganisation neu gedacht. Verlag systemisches Management VSM, Wien 2009. 50 Seiten. 14,90 Euro. ISBN 978-3902155-09-2. 
Demgegenüber wenden differenzierte, entwicklungsorientierte Organisationen viel Zeit für offizielle Kommunikation auf und entwickeln diese systematisch weiter. Sie machen ihre Kommunikation zum Gegenstand von Kommunikation. Die hierfür aufgewendete Zeit ist leicht messbar und zumeist einem hohen Legitimationsdruck ausgesetzt. Dies mag ein Grund sein, dass hoch effiziente, erprobte Großgruppenverfahren wie Open Space, Real Time Strategic Change (RTSC) oder Zukunftskonferenz, die raschen Wandel unter Einbeziehung vieler Menschen ermöglichen, selten eingesetzt werden.

Wie in der Erläuterung zu den Ergebnissen des Bürokratietests ausgeführt, gibt es klare Rahmenbedingungen, unter denen Bürokratien ausgezeichnet funktionieren. Ebenso gibt es eindeutige Kontra-Indikationen für bürokratische Organisationsformen.

In der Realität liegen jedoch zumeist gemischte Rahmenbedingungen vor. Es gibt für Organisationen ebenso Gründe, sich bürokratisch zu organisieren wie Gründe, sich partizipativ, ökologisch, projektförmig, an Prinzipien der Selbstorganisation orientiert zu formieren.

Typischerweise durchlaufen Organisationen nach Friedrich Glasl eine Entwicklungsgeschichte. Sie beginnen als PionierOrganisation, in der Teamgeist oder eine Gründer-Persönlichkeit als Koordinationsform genügen. Wenn dieses Modell nach erfolgreichem Wachstum an seine Grenzen stößt, ist Bürokratisierung angesagt: Aufbau eines beherrschbaren Apparates, Differenzierungsphase. Wenn sich dann zeigt, dass das gemeinsame Ganze verloren gegangen ist und die Organisation dysfunktional aufgespalten ist, gewinnen Vision und Leitbilder an Bedeutung, rücken die Aufgaben und Ergebnisse sowie die Umwelt und Anspruchsgruppen der Organisationen (wieder) in den Vordergrund: Entwickeln eines ganzheitlichen Systems, Vernetzung mit den Umwelten, Integrations-, Assoziationsphasen.

Eine Organisation wird umso erfolgreicher und überlebensfähiger sein, je mehr sie spezifische und angemessene Antworten für ihr Geschäft entwickelt und für das Umfeld, in dem sie sich bewegt. Zudem gilt es, auf Veränderungen in den Umwelten rechtzeitig und angemessen zu reagieren. Eine Organisation ist dann gut, wenn sie differenziert und entwicklungsfähig ist und somit eine unbürokratische Steuerung und Entwicklung auch bürokratischer Organisationsprinzipien und Verfahren zu leisten vermag, soweit diese notwendig sind.

Organisationen, die soziale Dienstleistungen erbringen, haben es im Regelfall mit Anspruchsgruppen (Stakeholdern) zu tun, deren Kalküle sich markant voneinander unterscheiden: Verwaltungen (Sozialbürokratien) in unterschiedlichen Entwicklungsstadien von traditionell zu (New) Public Management, politische Akteure mit kurzfristig wechselnden, häufig verschwommenen Zielsetzungen, Anspruchsberechtigte (Klienten), die an Lösungen interessiert sind, die ihre Bedürfnisse abdecken. Um überlebensfähig zu sein und einen gesellschaftlichen Nutzen zu stiften, müssen soziale Dienstleistungsorganisationen die Eigenlogiken aller relevanten Stakeholder verstehen, mit ihnen produktive Kommunikationen unterhalten können und deren Bedürfnisse abdecken oder aber auch klare Abgrenzungen gegenüber nicht zu befriedigenden Anliegen ziehen.

Je offener sich Sozialunternehmen diesen Herausforderungen stellen, je ernster sie ihre Stakeholdern nehmen, desto mehr importieren sie die gesellschaftlichen, ökonomischen und politischen Spannungsfelder, in deren Kontext sie operieren, in ihre innere Verfasstheit. Die Geschäftsführung, die unmittelbar vor allem mit Beamten und Politikern kommuniziert, entwickelt tendenziell andere mentale Modelle und Sprachhaltungen als die Mitarbeiterinnen und Mitarbeiter, die unmittelbar soziale Dienstleistungen erbringen.

Entsprechend den von den Geldgebern gestellten Anforderungen, aber auch aufgrund geänderter Prioritäten und Ausrich-

\section{"Bürokratien entstehen aus Angst vor Chaos und Kontrollverlust"}

tungen der Aufsichtsorgane haben viele soziale Träger in den letzten Jahren zunehmend Managementinstrumente, insbesondere in den Bereichen Leistungskataloge, Prozessbeschreibungen, Controlling und Qualitätssicherung eingeführt. Teilweise werden Ansprüche gestellt und Aufwendungen betrieben, die deutlich über das Ausmaß in vergleichbaren Profit-Unternehmen hinausgehen.

Es besteht die Gefahr, dass die Identität von sozialen Dienstleistern und die Arbeitswirklichkeiten der in den Kernprozessen tätigen Mitarbeiterinnen und Mitarbeitern sich schleichend so verändern, dass die Effizienz und Qualität der Dienstleistungen nicht zu-, sondern abnimmt und es zu Motivationsverlusten kommt.

Hilfreich ist es, das übliche Organigramm auf den Kopf zu stellen und ganz nach oben die Organisationseinheiten zu rücken, die unmittelbar mit dem Klienten arbeiten. Alles, was darunter einzuordnen ist, also Management- und Unterstützungsfunktionen haben in diesem Bild der Organisationen die Aufgabe, die nach außen ge- henden Dienstleistungen der Organisation zu unterstützen und zu gewährleisten. Im Vordergrund stehen die Kernprozesse, nicht die Management- und Unterstützungsprozesse.

Die Dienstleistungen sozialer Einrichtungen zielen darauf ab, die Lebensqualität von Klienten zu erhöhen und deren Anpassung an ihr soziales Umfeld zu erleichtern. In der Praxis sind die Mitarbeitenden sozialer Organisationen häufig mit Leid, verminderten Lebenschancen, Chaos, hartnäckigen Problemen und unerfüllbaren Erwartungen konfrontiert. Unterstützt sie die Organisation dabei, lässt die Organisation ihre Mitarbeiterinnen und Mitarbeiter im Stich oder behindert die Organisation sie sogar?

\section{Resümee: Was Verantwortliche leisten müssen}

Das Management sozialer Dienste besteht in einem Balanceakt zwischen Untersteuerung und Übersteuerung. Untersteuerung bedeutet zu wenig Klarheit über die Aufgabe, professionelle Standards, Prioritätensetzung und Erfolgskriterien und erhöhte Wahrscheinlichkeit von Burnout. Übersteuerung bedeutet die Dominanz von Formalismen, das Entschwinden der Hauptaufgabe und entfremdet die Mitarbeitenden ihrer Organisation.
Die Leitung sozialer Dienste hat die zentrale Aufgabe, die Unterschiede der Anspruchsgruppen und die Differenzierungen im Binnenbereich der Organisation achtsam zu balancieren und die daraus entstehende Identität der Organisation in Interaktion mit Anspruchsgruppen und MitarbeiterInnen weiterzuentwickeln.

Leistungserbringung und Arbeitsbeziehungen sind auf verschiedenen Ebenen zumindest jährlich zu reflektieren und weiterzuentwickeln. Neben Team-Klausuren bieten sich Gruppenveranstaltungen und Kundenparlamente an. Hierbei ist regelmäßig zu thematisieren:

- Wie viel Bürokratie im alten und neuen Gewand können, wollen und müssen wir uns leisten?

- Wie verhalten sich Kosten und Nutzen der von uns betriebenen Formalisierung, Standardisierung und Zergliederung von Aufgaben und Dienstleistungen?

- Was sind wir unseren Anspruchsgruppen an Berechenbarkeit und Präzision schuldig? Wie viel Flexibilität und Anpassungsfähigkeit erwarten sie von uns? 